\title{
Adaptação de um método de investigação do impacto da emoção na memória ${ }^{1}$
}

\author{
Carmem Beatriz Neufeld - Faculdade Assis Gurgacz? \\ Priscila Goergen Brust - Pontifícia Universidade Católica do Rio Grande do Sul \\ Lilian Milnitsky Stein - Pontifícia Universidade Católica do Rio Grande do Suß
}

\begin{abstract}
Resumo
O presente estudo apresenta uma adaptação dos instrumentos e procedimentos elaborados por Cahill, Prins, Weber e McGaugh para a avaliação dos efeitos da emoção nas distorções da memória. $O$ material visa oferecer à comunidade científica lusófona um instrumento valioso para investigação da memória e, a partir dessa adaptação, das falsas memórias. O experimento teve por finalidade testar a validade desta adaptação para a realidade brasileira, bem como a eficiência da avaliação das falsas memórias. A amostra foi composta por 138 estudantes universitários de diversos cursos de graduação e instituições de ensino superior particulares. O procedimento adaptado contém 11 slides, cujas imagens são condizentes com a realidade brasileira, e um teste auto-administrado de memória de reconhecimento. Os resultados sugerem uma adequação da adaptação dos instrumentos e procedimentos, encontrando um efeito potencializador da emoção sobre as memórias verdadeiras, principalmente para participantes do sexo masculino. Considerando os resultados obtidos, o procedimento adaptado é efetivo para medir o efeito da emoção na memória e suas distorções, e a emoção parece inocular a memória contra os efeitos da falsificação.

Palavras-chave: Memória; Emoção; Falsas memórias; Histórias.
\end{abstract}

\section{Adaptation of a method for studying the effect of emotion on memory}

\begin{abstract}
This study presents an adaptation of Cahill, Prins, Weber and McGaugh's instruments and procedures on the assessment of the effect of emotion on memory distortions. The study main purpose was to provide the lusophone scientific community with a valuable instrument for the study of memory and false memories. To test if the new version of the target materials (slides and narratives) and memory test were adequate to assess both true and false memories. The experiment was conducted with 138 college students from several private universities. The procedure consisted of eleven slides adapted to the Brazilian reality and a yes-no recognition memory test. The results suggested that both instruments and procedures were properly adapted. In addition, emotion tended to increase male participants true memory scores. The instrument was effective to measure the effect of emotion on memory distortions, and emotion seams to inoculate memory from distortions.
\end{abstract}

Keywords: Terms: Memory; Emotion; False memories; Stories.

\section{Introdução}

O estudo das distorções da memória tem sido foco de inúmeras pesquisas em psicologia, muitas das quais visam à avaliação dos possíveis impactos da emoção na memória (Pergher, Grassi-Oliveira, Ávila \& Stein, 2005; Robinson, Storbeck, Meier \& Kirkeby, 2004). Dentre as distorções mais estudadas, estão as falsas memórias, isto é, lembranças de eventos que, na realidade, não ocorreram (Stein \& Neufeld, 2001).

Uma das teorias que tem sido empregada para explicar tal fenômeno é a teoria do Traço Difuso (Brainerd \& Reyna, 2005). Para essa teoria, a memória é processada paralelamente por dois sistemas independentes: a memória literal e a de essência. A memória de essência é ampla, robusta e armazena somente as informações que representam o significado da experiência como um todo. Já a memória literal codifica as informações de forma precisa, de modo que os detalhes são registrados e armazenados de forma episódica, sendo, por isso, mais suscetível ao esquecimento e à interferência, se comparada à memória de essência (Neufeld \& Stein, 2001).

A elucidação da interação falsas memórias e emoção, no entanto, ainda não foi alcançada, já que resultados incongruentes são encontrados na literatura (Adolphs, Denburg \& Tranel, 2001; Santos \& Stein, 2006). A dificuldade de manipulação da variável emoção

\footnotetext{
${ }^{1}$ Apoio Financeiro CAPES - Faculdade Assis Gurgacz.

Endereço para correspondência:

2 E-mail: cneufeld@fag.edu.br

3 Avenida Ipiranga, 6.681 - Prédio 11 - Sala 933 - 90619-900 - Porto Alegre-RS - Telefone: (51) 3320-3500 - Ramal 7741

E-mail: lilian@pucrs.br
} 
é fator importante para explicar tais resultados (Harris \& Pashler, 2006). No entanto, a compreensão da interface da emoção com as falsas memórias se faz necessária à medida que apresenta implicações práticas em diversas áreas das ciências da saúde (Loftus, 1997).

Esses estudos têm sua motivação em críticas encontradas na literatura de que tarefas excessivamente fáceis não utilizariam recursos atencionais suficientes para serem memoráveis, gerando assim um decréscimo na precisão da memória para eventos cotidianos e menos importantes. As falsas memórias, portanto, se restringiriam a fatos corriqueiros da vida das pessoas (Hudson, Fivush \& Kuebli, 1992). Sendo assim, para esses teóricos, as memórias relacionadas a situações traumáticas ou emocionalmente carregadas estariam imunes a tais erros de memória.

Em busca de esclarecer essa questão e de responder a tais críticas, alguns autores (Pergher, Stein \& Wainer, 2004; Wessel, Van der Kooy \& Merckelbach, 2000) têm demonstrado que a freqüência de memórias intrusivas e o evitar recordações do trauma indicam uma especificidade diminuída da memória autobiográfica, ou seja, que pessoas expostas a situações traumáticas tendem a apresentar falhas na memória para o evento. Tais achados sustentam, portanto, a conjetura de que a memória para eventos emocionalmente intensos, como ideação suicida, depressão severa, abuso sexual e guerra, é passível de distorção (Klimes-Dougan, 1998; LaBar \& Cabeza, 2006).

Esse fato possui implicações clínicas significativas quanto a distorções cognitivas e afetivas (Pergher e colaboradores, 2005), uma vez que as sessões terapêuticas giram em torno de experiências emocionalmente significativas vivenciadas pelo paciente. Nos últimos anos, diversos casos relatados na literatura de recuperação errônea de lembranças baseadas em procedimentos de terapeutas têm preocupado os pesquisadores (Andrews e colaboradores, 1999). Portanto observa-se a importância do estudo dos mecanismos envolvidos na recuperação de memórias com o intuito de auxiliar o desenvolvimento e aprimoramento de técnicas de entrevista e de intervenção terapêutica que minimizem a ocorrência ou o impacto dos erros de memória.

Uma das contribuições para os estudos de emoção e memória foi a produção, por Cahill, Prins, Weber e McGaugh (1994), de um procedimento capaz de testar a influência da emoção sobre a memória. O material original foi uma réplica dos estudos de Heuer e Reisberg (1990) e consistia de 12 slides e 12 frases narrando a história retratada nos slides em duas versões. Em um aprimoramento do material Cahill e McGaugh (1995) retiraram um dos slides e refizeram os outros, adaptando-os a realidade local. O material totalizou 11 slides e duas versões de 11 frases narrativas.

A primeira versão da história, chamada de versão neutra, narrava o passeio de um menino com sua mãe até o local de trabalho de seu pai. No caminho, o menino passava por um carro acidentado, e no hospital ele assistia a um treinamento de emergência. A versão denominada emocional relatava que durante o passeio o menino sofria um acidente, causando-lhe graves ferimentos. Os slides foram divididos em três fases distintas. As fases inicial e final das duas versões eram idênticas, diferenciando-se apenas a narrativa da fase intermediária, onde uma das versões foi considerada mais carregada emocionalmente (acidente envolvendo o menino) do que a outra. Além disto, o procedimento consistia também de um teste de memória de múltipla escolha com, em média, 6 questões para cada um dos slides. Cada questão apresentava quatro opções de resposta, sendo o participante instruído a escolher apenas uma.

O foco das pesquisas que utilizam esse material concentra-se na investigação do papel da amígdala no processamento de estímulos altamente emocionais (Cahill \& McGaugh, 1995). As evidências neuroanatômicas sugerem que a amígdala participa no controle automático das respostas eliciadas por estímulos emocionalmente salientes, embora pouco se conheça sobre seu funcionamento em tal controle. A hipótese é a de que o nível de emocionalidade produzido por um estímulo determina o nível de participação da amígdala no armazenamento da memória para tal.

O material foi traduzido para o português por Quevedo e colaboradores (2003) e utilizado em pesquisas no Brasil com os mesmos slides, mantendo o mesmo padrão norte-americano de apresentação. A ênfase dessas pesquisas foi a observação da consolidação da memória nos sistemas de curto e longo prazo, apontando este último como mais suscetível ao efeito da emoção do que o primeiro, achados esses que corroboram aqueles encontrados por McGaugh (2000).

Observam-se, no entanto, limitações inerentes ao material original, que data de estudos antigos e culturalmente distintos, o que nos motivou a adaptar o material pictográfico para a realidade brasileira. Além disso, simplificamos o teste de memória de recordação com pistas, transformando-o em teste de reconhecimento e acrescentando medidas de falsas memórias. A inclusão da medida de falsas memórias no material é uma inovação que visa suprir a falta de subsídios para o esclarecimento das posições distintas sobre a falsificação da memória para eventos emocionalmente intensos.

Para tanto, foram conduzidos dois estudos. O primeiro descreve minuciosamente o processo de adaptação do material, incluindo a avaliação da Psico-USF, v. 13, n. 1, p. 21-29, jan./jun. 2008 
emocionalidade dos novos slides. $O$ segundo estudo deteve-se na avaliação da fidedignidade do material, buscando produzir resultados semelhantemente válidos para a avaliação da influência da emoção na memória, bem como nas falsas memórias.

\section{Método}

\section{Estudo 1}

O Estudo 1 envolve as etapas da adaptação do procedimento de investigação de memória e emoção de Cahill e colaboradores (1994), aprimorado por Cahill e McGaugh (1995), e traduzido para o português por Quevedo e colaboradores (2003).

\section{Participantes}

A amostra foi composta por estudantes universitários pertencentes ao curso de graduação em psicologia de uma instituição privada de ensino superior do Rio Grande do Sul. Os participantes foram selecionados por conveniência e distribuídos de acordo com o objetivo de cada etapa da adaptação, nas quais serão mais precisamente descritos.

\section{Instrumentos}

O material original era composto de uma seqüência de 11 slides, duas versões de frases que relatavam duas histórias e um teste de memória. Neste trabalho, as versões das histórias serão denominadas controle e experimental, respectivamente, pois se entende que nenhuma das versões poderia ser considerada neutra, apenas de apelo emocional menos intenso. A tradução das frases e do teste de memória para o português já havia sido feita por Quevedo e colaboradores (2003); no entanto, foram necessárias novas adaptações para utilização dos instrumentos na testagem de falsas memórias. Essas adaptações foram realizadas em diversas etapas que serão apresentadas a seguir.

\section{Procedimentos}

Etapa 1. Inicialmente, foi realizado um estudo piloto para testar a emocionalidade que seria atribuída ao material, em uma escala subjetiva de emoção, já que o material original lançava mão de medidas fisiológicas de emoção que não foram viabilizadas no presente estudo. Após a assinatura do Termo de Consentimento Livre e Esclarecido, apresentaram-se os slides e a história a oito estudantes universitários, que foram questionados se o material havia despertado alguma emoção e, em caso de resposta afirmativa, foram solicitados a apontar o nível da intensidade da emoção sentida em uma escala de 1 (muito pouco) a 5 (extrema). Os participantes julgaram o material pouco condizente com a realidade brasileira e destacaram que, apesar da história do acidente do Psico-USF, v. 13, n. 1, p. 21-29, jan./jun. 2008 menino (experimental) poder ser considerada comovente, sua forma de apresentação (as imagens dos slides) não chegava a despertar emoção significativa. Além disso, foi apontado que a qualidade dos slides dificultava sua visualização, impossibilitando até mesmo a identificação de alguns detalhes do material original.

Etapa 2. Com base nos resultados obtidos na etapa 1, foram produzidos novos slides com intuito, além de aprimorar a qualidade das imagens, de buscar uma maior adequação à realidade brasileira. Tal proposta justifica-se não apenas pela não-adequação das imagens retratadas nos slides à realidade brasileira, mas também pelo tempo transcorrido entre a produção do material original (final da década de 80) e o momento atual, já que ocorreram inúmeras inovações tecnológicas que potencializaram a clareza e fidedignidade de imagens. Portanto, do material original utilizado por Cahill e colaboradores (1994) e traduzido por Quevedo e colaboradores (2003), foi mantido apenas o slide crítico (slide 8), que retrata os pés reimplantados do menino após $\mathrm{o}$ acidente. As demais fotos foram todas substituídas.

As fotos para produção dos slides que retratavam a mãe e o menino foram produzidas em uma cidade do interior do Rio Grande do Sul. Foi concedida autorização por escrito dos responsáveis pela criança que protagonizou o menino, bem como da participante que representava a mãe, para utilização e veiculação das imagens para fins de pesquisa científica, sem qualquer limitação de número de inserções e reproduções na seqüência de slides. Os slides que representam o pai, o carro acidentado e os procedimentos hospitalares foram retirados de bancos de dados de domínio público na rede mundial de computadores (Allposters.com, 2004). O novo material manteve as mesmas características do material original em termos de personagens, objetos, ações e ambiente, ou seja, onde havia uma mãe e seu filho caminhando, procurou-se manter as mesmas disposições de espaço e semelhança de personagens.

Etapa 3. Após a finalização da nova versão da seqüência de slides foi conduzido um novo estudo piloto com intuito de avaliar a adequação dos slides acompanhados das narrativas experimental e controle. As duas versões da história foram gravadas em $\mathrm{CD}$, narradas por uma acadêmica em fase de conclusão do curso de comunicação social, com o cuidado de manter fixo o intervalo de 6s para a apresentação de cada slide.

Após a assinatura do Termo de Consentimento Livre e Esclarecido, o material foi apresentado para 10 estudantes universitários, aos quais foi solicitado que avaliassem a intensidade (de 0 a 5 ) da emoção despertada a partir do mesmo. Os estudantes que receberam a versão experimental consideraram o material com intensidade emocional moderada (níveis entre 2 e 3). Já 
os participantes que ouviram a versão controle apontaram níveis muito baixos de intensidade da emoção (entre 0 e 1$)$.

Etapa 4. A partir dos resultados da etapa anterior, deu-se início à produção do teste de memória de reconhecimento. O teste de memória de Cahill e colaboradores (1994), traduzido para o português por Quevedo e colaboradores (2003), consistia de 76 questões de múltipla escolha com 4 opções de resposta. Com intuito de adaptar o material para testar falsas memórias, foi necessária a produção de um novo teste.

Foram formuladas 31 frases para compor o teste de memória de reconhecimento. As frases-alvo eram afirmativas, condizentes com a seqüência de slides ou com a narrativa apresentada e foram propostas com o objetivo de ser a medida de memórias verdadeiras dos participantes (por exemplo, a mãe tranca a porta antes de sair de casa). As frases relacionadas eram afirmativas incorretas, porém que preservavam a mesma essência semântica do alvo (por exemplo, na seqüência de slides aparece um carro acidentado de cor azul [alvo]; um item relacionado poderia ser uma afirmativa no teste de que o carro do acidente era vermelho). As frases relacionadas foram apresentadas tendo em vista possibilitar a medida de falsas memórias dos participantes. Já a apresentação das frases nãorelacionadas (por exemplo, a tia do menino trabalha em um escritório) tinha como objetivo principal atuar como medida de resposta de viés ou "chute" dos participantes, já que essas frases não apresentavam relação alguma com a seqüência de slides ou com a história narrada e, portanto, sua aceitação no teste de reconhecimento possivelmente não teria uma base mnemônica.

Dentre as 31 frases, 10 foram consideradas frases-alvo; 12, frases relacionadas e 9, frases nãorelacionadas. Como o material-alvo (seqüência de slides e narrativas) era dividido em três fases distintas, para fase 1 foram propostas três frases-alvo, quatro frases relacionadas e três frases não-relacionadas. Já para a fase 2 , em que as versões da história se diferenciam em experimental e controle, foram propostas quatro frasesalvo para o teste experimental e quatro outras frasesalvo para o teste controle. As quatro frases relacionadas e as três frases não-relacionadas foram as mesmas em ambos os testes. Para a fase 3, foram propostas três frases-alvo, quatro frases relacionadas e três frases nãorelacionadas. A ordem de distribuição das frases no teste foi aleatória, com o cuidado de que as frases-alvo da fase 2 dos testes de memória controle e experimental mantivessem a mesma numeração em ambos os testes.

Etapa 5 e 6. Com intuito de testar a adequação do teste de memória foi realizado um estudo piloto com dois grupos de participantes, cada um com 20 estudantes universitários, aos quais foi apresentada a seqüência de slides e uma das versões da história (experimental ou controle). Em seguida, foi solicitado que classificassem a intensidade da emoção despertada pela história em uma escala de 0 a 5 . Por fim, foi aplicado o teste de memória condizente com a versão que o participante assistiu.

A partir deste estudo, algumas alterações foram necessárias nos instrumentos desenvolvidos. Em um teste de memória imediato, não são esperados níveis médios elevados de falsas memórias e de respostas de viés. No entanto, foram identificadas algumas frases relacionadas (medida de falsas memórias) e nãorelacionadas (medidas de resposta de viés) com índices médios muito elevados, denotando uma não-adequação das mesmas ao teste, tendo sido excluídas. Sendo assim, a versão definitiva do teste de memória de reconhecimento auto-administrado foi composta de 25 frases.

Em suma, foi aí completada a etapa de adaptação do material e do teste de reconhecimento para a realidade brasileira e para a avaliação das falsas memórias. O material-alvo, então, ficou composto de uma seqüência de 11 slides acompanhados por narrativas coerentes, divididos em três fases. A fase 1 (slides de 1 a 4) apresentava uma mãe e seu filho na rua, a caminho do hospital, e a foto do pai em um laboratório, a fase 2 (slides de 5 a 8) apresentava um carro batido e diversos procedimentos hospitalares, já a fase 3 (slides de 9 a 11) apresentava a mãe caminhando, ao telefone e indo embora. O teste de memória de reconhecimento ficou assim distribuído: 10 itens-alvo (três referentes à fase 1, quatro à fase 2 e três à fase 3), 6 itens distratores relacionados (dois itens para cada fase), e 9 itens nãorelacionados.

\section{Método}

\section{Estudo 2}

O estudo 2 envolveu um delineamento experimental fatorial misto $2 \times 2 \times 3 \times 3$ com medidas repetidas nas duas últimas variáveis. A primeira variável, nível de emocionalidade do material-alvo, foi manipulada entre grupos. Como essa variável apresentava dois níveis, havia dois grupos de participantes que receberam diferentes tratamentos. $\mathrm{O}$ grupo experimental recebeu o material-alvo que relatava o acidente do menino (considerado com maior carga emocional), enquanto o grupo controle recebeu o material-alvo que relatava apenas a visita do menino ao hospital (representando carga emocional desprezível). A variável sexo ficou distribuída homogeneamente entre os dois grupos. Todos os participantes receberam 
material-alvo dividido em três fases e três tipos de itens no teste de memória (alvo, relacionado e nãorelacionado). As variáveis dependentes foram o desempenho da memória e a atribuição subjetiva do nível de emoção despertada pelo material-alvo.

\section{Participantes}

A amostra foi composta por 138 estudantes universitários, selecionados por conveniência, e pertencentes a diversos cursos de graduação (administração, engenharia, pedagogia, psicologia, enfermagem) de uma instituição privada de ensino superior do Paraná, com idades entre 17 e 32 anos $(M=20,77, D P=3,58)$. Os participantes foram designados aleatoriamente para um dos dois grupos, experimental ou controle. O grupo experimental foi composto de 58 participantes (30 eram homens), enquanto 80 estudantes compuseram o grupo controle (34 eram homens).

\section{Instrumentos}

O material utilizado no presente estudo corresponde àquele da adaptação anteriormente descrita, composto de uma seqüência de 11 slides divididos em três fases. Para cada slide havia uma frase narrando o acontecimento. Os participantes foram aleatoriamente divididos em dois grupos de acordo com a apresentação da narrativa: emocionalmente carregada ou não. Ou seja, tanto o grupo experimental quanto o grupo controle receberam a mesma seqüência de slides; no entanto, recebiam versões distintas das narrativas da história.

O teste de memória de reconhecimento autoadministrado foi composto pelos 25 itens elaborados no estudo 1. A escala subjetiva de emoção consistia de duas questões. A primeira foi "A história que você acabou de ouvir causou alguma emoção em você?", com opções "sim" e "não", conforme a escolha do participante. A segunda dizia: "Em caso afirmativo, marque um $\mathrm{X}$ no número correspondente ao nível de intensidade da emoção que você sentiu ao ouvir a história e ver os slides apresentados anteriormente", e era seguida por uma tabela com uma escala Likert de 5 pontos, variando de "quase nenhuma" (1) até "extrema" (5).

\section{Procedimentos}

A coleta de dados foi realizada em grupo, em sala silenciosa, com a utilização de equipamento de multimídia para projeção dos slides a partir do Power Point for Windows XP e aparelho de som para reprodução do CD, com a versão experimental ou controle da história. Após uma explicação do estudo e de assegurada a total liberdade para o(a) participante desistir a qualquer momento da pesquisa, os voluntários assinaram um Termo de Consentimento Livre e Esclarecido, elaborado consoante as diretrizes éticas que regulamentam a pesquisa com seres humanos. Inicialmente foi dito aos participantes que o estudo envolvia a coleta da opinião de estudantes universitários sobre diferentes histórias, não thes tendo sido avisado que sua memória seria testada. Apenas foi solicitado aos participantes que prestassem bastante atenção na seqüência de slides e na história que seria apresentada, frisando a importância dos mesmos evitarem fazer comentários ao longo dos procedimentos para não influenciarem as opiniões uns dos outros.

Logo após a apresentação do material-alvo foi entregue aos participantes a escala subjetiva de emoção, sendo-lhes solicitado responder sobre a intensidade que atribuíam à emoção despertada pelo material-alvo. Por fim, foi aplicado o teste imediato de memória de reconhecimento.

Os participantes foram cuidadosamente instruídos a marcar a opção "sim" sempre que a frase correspondesse ao que foi apresentado no material-alvo. Foi dito aos participantes: "Baseie suas respostas apenas na sua memória dos slides que viu e do relato da história que ouviu. Marque 'não' para todas as opções que não sejam as que você viu ou ouviu, mesmo que sejam coisas que você possa inferir a respeito do evento". Foi apresentado um item exemplo para treinamento dos participantes e solicitado que as questões fossem respondidas na ordem em que aparecessem no teste, não deixando nenhuma questão em branco, nem retornando às questões anteriores. A experimentadora frisou ainda a importância de ler as instruções com atenção e de permanecer em silêncio após o término do teste.

\section{Resultados}

Todas as informações coletadas foram armazenadas em um banco de dados elaborado especialmente para este fim e analisados com o auxílio do programa Statistical Package for Social Sciences (SPSS), versão 12.0. Todos os tratamentos estatísticos utilizaram um $a<0,05$ para os testes de hipóteses. Foram realizadas análises post hoc e comparações pareadas com correção de Bonferroni.

\section{Indicadores de emocionalidade}

Os resultados da escala subjetiva de emoção (escala Likert de 5 pontos) foram testados por meio de uma ANOVA para grupo (experimental vs. controle) $\mathrm{x}$ sexo (masculino vs. feminino). Nessa análise, foi evidenciado efeito principal de grupo $(\mathrm{F}(1,134)=$ 40,356, $\mathrm{p}<0,001)$ e de sexo $(\mathrm{F}(1,134)=6,736, \mathrm{p}<0,05)$.

No que tange à comparação entre o grupo experimental e controle, em relação ao nível de emocionalidade atribuída na escala subjetiva de emoção, os participantes do grupo experimental $(\mathrm{M}=2,34, \mathrm{DP}=$ 
1,45) avaliaram o material-alvo com maior intensidade emocional do que os participantes do grupo controle $(\mathrm{M}=0,95, \mathrm{DP}=1,05)$.

Esses resultados sugerem que o material-alvo experimental despertou uma maior intensidade de emoção do que o controle. Apesar do material-alvo não ter sido produzido e testado com medidas fisiológicas como em outros estudos com material semelhante (Cahill e colaboradores, 1994; Robinson e colaboradores, 2004), os resultados indicam que a avaliação subjetiva da emocionalidade pelos participantes foi afetada pela manipulação da variável emoção através das distintas versões das narrativas. Tal resultado corrobora os estudos de Lang, Greenwald, Bradley e Hamm (1993), que afirmam haver uma relação entre medidas subjetivas de emoção e medidas fisiológicas.

No que se refere ao efeito da variável sexo sobre a avaliação subjetiva da emocionalidade do material-alvo, observou-se que, de forma geral, os homens consideraram o material-alvo com maior carga emocional ( $\mathrm{M}=1,91$, $\mathrm{DP}=1,433)$ do que as mulheres $(\mathrm{M}=1,22, \mathrm{DP}=1,327)$ $(\mathrm{F}(1,136)=0,756, \mathrm{p}<0,005)$. Destaca-se aqui que essa diferença se refere somente ao efeito principal da variável, e que não houve interação entre as variáveis grupo e sexo.

\section{Indicadores de memória}

A avaliação do desempenho da memória dos participantes foi baseada em três tipos de respostas no teste de memória: $\mathrm{MV}=$ memórias verdadeiras (respostas "sim" para os itens-alvo); FM=falsas memórias (respostas "sim" para os itens semanticamente relacionados); e $\mathrm{RV}=$ respostas de viés (respostas "sim" para os itens não relacionados semanticamente).

Considerando os resultados de memória geral, as respostas para o teste de memória foram analisadas por meio de uma ANOVA para grupo (experimental vs. controle) x sexo (masculino vs. feminino) $\mathrm{x}$ item (alvo $v s$. itens relacionados $v s$. itens não-relacionados), com medidas repetidas na última variável.

Esta ANOVA evidenciou efeito principal de item $(F(2,134)=2728,640, p<0,001)$. Foi encontrado um alto índice de $\mathrm{MV}(\mathrm{M}=0,802, \mathrm{DP}=0,129)$, um menor índice de FM $(\mathrm{M}=0,222, \mathrm{DP}=0,208)$ e um índice mínimo de RV (M 0,016, DP=0,043), sendo estas diferenças estatisticamente significativas ( $\mathrm{ps}<0,001$ ).

O efeito principal da ANOVA foi qualificado por uma interação entre item e sexo $(F(2,134)=9,576$, $\mathrm{p}<0,001)$. Considerando os índices de MV $(\mathrm{F}(1,134)=15,197, \mathrm{p}<0,001)$, os homens obtiveram índices superiores $(M=0,843, D P=0,097)$ aos das mulheres $(\mathrm{M}=0,761, \mathrm{DP}=0,140)$. Em relação aos índices de FM e de RV não foram observadas diferenças significativas entre sexo $(\mathrm{Fs}(1,134)<1,321$, ps $>0,05)$.

Os resultados do teste de memória foram analisados por meio de uma ANOVA para grupo (experimental vs. controle) $\mathrm{x}$ sexo (masculino vs. feminino) $\mathrm{x}$ fase (fase $1 v$ s. fase $2 v$ s. fase 3 ) $\mathrm{x}$ item (alvo vs. item relacionado vs. item não-relacionado), com medidas repetidas nas duas últimas variáveis.

A partir desta ANOVA, três efeitos principais foram evidenciados: item $(\mathrm{F}(2,136)=2026,387$, $\mathrm{p}<0,001)$, fase $(\mathrm{F}(2,136)=47,375, \mathrm{p}<0,001)$ e sexo $(\mathrm{F}(1,134)=7,106, \mathrm{p}<0,01)$. Estes efeitos principais foram qualificados por interações entre fase e grupo $(\mathrm{F}(2,136)=4,311, \mathrm{p}<0,05)$; item e fase $(\mathrm{F}(2,136)=68,683$, $\mathrm{p}<0,001)$; item e sexo $(\mathrm{F}(2,136)=8,701, \mathrm{p}<0,001)$. Também houve interação entre as variáveis item, fase e grupo $(\mathrm{F}(2,136)=4,254, \mathrm{p}<0,01)$, conforme pode se observar na Tabela 1.

Tabela 1 - Indicadores de memória: média de reconhecimento por tipo de item, por fase e por grupo

\begin{tabular}{cllcc}
\hline & & & Item & \\
Fase & \multicolumn{1}{c}{ Grupo } & MV & FM & RV \\
\hline 1 & Experimental & $0,701(0,297)$ & $0,344(0,353)$ & $0,022(0,084)$ \\
& Controle & $0,696(0,259)$ & $0,362(0,355)$ & $0,016(0,072)$ \\
2 & Experimental & $0,991(0,046)^{* *}$ & $0,189(0,244)$ & $0,011(0,061)$ \\
& Controle & $0,912(0,132)^{* *}$ & $0,200(0,314)$ & $0,033(0,100)$ \\
3 & Experimental & $0,696(0,236)$ & $0,017(0,092)^{* *}$ & 0,0 \\
& Controle & $0,692(0,230)$ & $0,187(0,311)^{* *}$ & $0,008(0,052)$ \\
\hline
\end{tabular}

Nota. Desvio padrão entre parênteses

$\mathrm{MV}=$ memórias verdadeiras; $\mathrm{FM}=$ falsas memórias; $\mathrm{RV}=$ respostas de viés

$* \mathrm{p}<0,05$

$* * \mathrm{p}<0,001$ 
Comparando os índices do grupo experimental e controle de acordo com a fase (Tabela 1), foram evidenciadas diferenças significativas para MV na fase 2, tendo o grupo experimental obtido índices superiores ao do grupo controle $(\mathrm{F}(1,136)=18,865, \mathrm{p}<0,001)$. No que se refere às FM foi detectado um maior índice de FM para $\mathrm{o}$ grupo controle do que para o grupo experimental na fase 3 $(\mathrm{F}(1,136)=16,224, \mathrm{p}<0,001)$.

No grupo experimental houve uma maior ocorrência de MV na fase $2(\mathrm{~F}(1,136)=57,062, \mathrm{p}<0,001)$, não sendo detectada diferença significativa nos índices de MV entre a fase 1 e a fase $3(F(1,136)=57,062, p>0,05)$. Em relação às FM ocorreu um decréscimo nos índices ao longo do experimento, tendo os mesmos alcançado os níveis mais elevados na fase 1 , decrescendo na fase 2 e subseqüentemente na fase $3(\operatorname{Fs}(1,136)=20,236$, ps $<$ $0,001)$. Já as RV, que apresentavam baixos níveis de aceitação na fase 1 , obtiveram índice nulo na fase 3 $(\mathrm{F}(1,136)=3,774, \mathrm{p}<0,05)$.

Quando consideradas as médias do grupo controle, os índices de MV foram semelhantes aos relatados para o grupo experimental, onde a fase 2 obteve os maiores índices $(\mathrm{F}(1,136)=43,831, \mathrm{p}<0,001)$. Já no que se refere aos índices de FM, ocorreu um decréscimo dos índices da fase 1 para a fase $2(F(1,136)=10,105, \mathrm{p}<0,001)$; no entanto, não houve diferença entre as fases 2 e 3 ( $p>$ $0,05)$. As RV mantiveram índices muito baixos constantes ao longo das três fases $(\mathrm{F}(1,136)=3,334, \mathrm{p}>0,05)$.

\section{Discussão}

Tomando por base os resultados, no que tange aos indicadores de emocionalidade, o material-alvo experimental despertou maior intensidade de emoção do que o controle. Apesar do material-alvo não ter sido produzido e testado com medidas fisiológicas como em outros estudos com material semelhante (Cahill e colaboradores, 1994; Robinson e colaboradores, 2004), os resultados indicam que a avaliação subjetiva da emocionalidade pelos participantes foi afetada pela manipulação da variável emoção através das distintas versões das narrativas. Tal resultado corrobora os estudos de Lang, Greenwald, Bradley e Hamm (1993) que afirmam haver relação entre medidas subjetivas de emoção e medidas fisiológicas.

Considerando os indicadores de memória, os resultados sugerem uma adequação dos itens do teste de memória e uma boa qualidade da memória dos participantes, visto que nos dados apontados pela literatura (Cahill \& Andreano, 2006) alvos geralmente são mais aceitos do que itens relacionados, e itens relacionados são mais aceitos do que itens não-relacionados.

Já no que se refere às médias superiores de memórias verdadeiras entre os homens em relação as mulheres, tal resultado corrobora as evidências recentemente destacadas por Cahill e Andreano (2006). Os autores referem que, nas questões sobre eventos emocionais, o desempenho no teste de reconhecimento de múltipla-escolha desenvolvido por Cahill e colaboradores (1994) foi superior em homens com relação a informações centrais do evento. Já as mulheres apresentaram um aumento significativo na recuperação de detalhes. No teste de memória desenvolvido para o presente estudo, os itens versavam primordialmente sobre informações centrais ao evento, sendo apresentadas poucas questões baseadas em detalhes periféricos da história. Nesse sentido, o teste de memória parece ter favorecido o índice de reconhecimento dos homens, que talvez esteja diretamente relacionado com sua capacidade de recordar eventos centrais da história (Cahill \& Van-Stegeren, 2003).

No que tange à comparação entre grupo experimental e controle os resultados sugerem um efeito potencializador da emoção sobre as MV, já que a fase 2 pode ser considerada a parte mais emocionalmente carregada do evento. A retenção de informações centrais da fase 2, no entanto, foi significativamente maior do que para a fase 1 , o que também torna os dados consistentes com a literatura, visto que há um aumento do índice de recordação para informações centrais associadas com uma situação emocionalmente carregada (Kern, Libkuman, Otani \& Holmes, 2005).

Além disso, os índices de FM foram superiores no grupo controle, sugerindo que a emoção em níveis moderados serve como inoculador das $\mathrm{MV}$, ocorrendo um decréscimo das FM. Essa hipótese parece corroborar a idéia de Mandler (1975) e de Robinson e colaboradores (2004) de que, em situações com uma carga emocional leve a moderada, a memória dos participantes parece ser auxiliada graças aos recursos adicionais de atenção deslocados para o processamento do evento.

\section{Considerações finais}

O presente estudo objetivou oferecer à comunidade científica brasileira instrumentos e procedimentos de avaliação de memória e emoção, como também, de investigação do efeito da emocionalidade sobre as falsas memórias. O estudo dos mecanismos envolvidos neste processo pode auxiliar no desenvolvimento e aprimoramento de técnicas de entrevista e de intervenção terapêutica que minimizem a ocorrência ou o impacto dos erros de memória, por exemplo.

Assim sendo, tomou-se por base o procedimento de Cahill e colaboradores (1994), já utilizado em diversos idiomas, além do original em inglês, como o alemão 
(Cahill, Babinsky, Marlowitsch \& McGaugh, 1995), japonês (Kazui e colaboradores, 2000), italiano (Gasbarri e colaboradores, 2006), e também o português (Quevedo e colaboradores, 2003), procedendo à adaptação para o Brasil e preservando as características essenciais da versão original.

No entanto, o procedimento adaptado e descrito acima não se restringe à investigação de falsas memórias em adultos saudáveis. O desafio encontra-se em testar sua adequação em diferentes populações como, por exemplo, com grupos clínicos, em indivíduos com diferentes graus de escolaridade, e em diferentes fases do desenvolvimento (como adultos jovens e idosos). Assim sendo, refinamentos metodológicos podem prover dados interessantes. Dentre eles, podem ser citadas a testagem da memória por recordação, ao invés de reconhecimento, e a inserção de material de sugestão de falsa informação.

Além disso, algumas limitações podem ser apontadas no presente estudo. Uma delas é o fato dos itens do teste estarem direcionados para informações centrais do evento, o que parece ter favorecido as lembranças verdadeiras dos homens acerca da história. Faz-se necessária, então, a inserção de detalhes periféricos ao evento nos itens do teste, para que se possa comparar o desempenho da memória para detalhes centrais e periféricos.

\section{Referências}

Adolphs, R., Denburg, N. L. \& Tranel, D. (2001). The amygdala's role in long-term declarative memory for gist and detail. Behavioral Neuroscience, 15(5), 983-992.

Allposters.com (2004). Obtido do World Wide Web: www.allposters.com/gallery.asp. Berkeley, California, USA.

Andrews, B., Brewin, C. R., Ochera, J., Morton, J., Bekerian, D. A., Davies, G. M. \& Mollon, P. (1999). Characteristics, context and consequences of memory recovery among adults in therapy. British Journal of Psychiatry, 175, 141-146.

Brainerd, C. J. \& Reyna, V. F. (2005). The science of false memory. Nova Iorque: Oxford University Press.

Cahill, L. \& McGaugh, J. L. (1995). A novel demonstration of enhanced memory associated with emotional arousal. Consciousness and Cognition, 4, 410-421.

Cahill, L. \& Van-Stegeren, A. (2003). Sex-related impairment of memory for emotional envents with beta-adrenergic blockade. Neurobiology of Learning and Memory, 79(1), 81-88.
Cahill, L. \& Andreano, J. M. (2006). Glucocorticoid release and memory consolidation in men and women. Psychological Science, 17(6), 466-470.

Cahill, L., Babinsky, R., Marlowitsch, H. J. \& McGaugh, J. L. (1995). The amygdala and emotional memory. Nature, 377, 295-296.

Cahill, L., Prins, B., Weber, M. \& McGaugh, J. L. (1994). Beta-adrenergic activation and memory for emotional events. Nature, 371, 702-704.

Gasbarri, A., Arnone, B., Pompili, A., Marchetti, A., Pacitti, F., Calil, S. S., Pacitti, C., Tavares, M. C. \& Tomaz, C. (2006). Sex-related lateralized effect of emotional content on declarative memory: An event related potential study. Behavioural Brain Research, 168 , 177-184.

Harris, C. R. \& Pashler, H. (2006). Enhanced memory for negatively emotionally charged pictures without selective rumination. Emotion, 5(2), 191-199.

Heuer F. \& Reisberg D. (1990). Vivid memories of emotional events: The accuracy of remembered minutiae. Memory and Cognition, 18(5), 496-506.

Hudson, J., Fivush, R. \& Kuebli, J. (1992). Scripts and episodes: The development of event memory. Applied Cognitive Psychology, 6, 483-505.

Kazui, H., Mori, E., Hashimoto, M., Hirono, N., Imamura, T., Tanimukai, S., Hanihara, T. \& Cahill, L. (2000). Impact of emotion on memory: Controlled study of the influence of emotionally charged material on declarative memory in Alzheimer's disease. British Journal of Psychiatry, 177, 343-347.

Kern, R. P. K., Libkuman, T. M., Otani, H. \& Holmes, K. (2005). Emotional stimuli, divided attention, and memory. Emotion, 5(4), 408-417.

Klimes-Dougan, B. (1998). Screening for suicidal ideation in children and adolescents: Methodological considerations. Journal of Adolescence, 21(4), 435-444.

LaBar, K. S. \& Cabeza, R. (2006). Cognitive neuroscience of emotional memory. Nature, 7, 54-64.

Lang, P. J., Greenwald, M. K., Bradley, M. M. \& Hamm, A. (1993). Looking at pictures: Affective, facial, visceral, and behavioral reactions. Psychophysiology, 30, 261-273.

Loftus, E. F. (1997). Creating false memories. Scientific American, 277, 70-75.

Mandler, G. (1975). Mind and emotion. Em G. Mandler (Org.). Mind and Emotion (p. 83-110). Nova Iorque: John Wiley \& Sons. 
McGaugh, J. L. (2000). Memory: A century of consolidation. Science, 287(5451), 248-251.

Neufeld, C. B. \& Stein, L. M. (2001). A compreensão da memória segundo diferentes perspectivas teóricas. Revista Estudos de Psicologia, 18(2), 50-63.

Nie, N. H., Hull, C. H. \& Bent, D. H. (2003). Statistical package for social sciences (Versão 12.0) [Software computacional]. São Paulo, SP: SPSS Inc.

Pergher, G. K., Stein, L. M. \& Wainer, R. (2004). Estudos sobre a memória na depressão: achados e implicações para a terapia cognitiva. Revista de Psiquiatria Clínica, 31(2), 82-90.

Pergher, G. K., Grassi-Oliveira, R., Ávila, L. M. \& Stein, L. M. (2005). Memória, humor e emoção. Revista de Psiquiatria do Rio Grande do Sul, 28(1), 5-12.

Quevedo, J., Sant'Anna, M. K., Madruga, M., Lovato, I., de-Paris, F., Kapczinski, F., Izquierdo, I. \& Cahill, L. (2003). Differential effects of emotional arousal in short and long-term memory in healthy adults. Neurobiology of Learning and Memory, 79(132-135).
Robinson, M. D., Storbeck, J., Meier, B. P. \& Kirkeby, B. S. (2004). Watch out! That could be dangerous: Valence-arousal interactions in evaluative processing. Personality and Social Psychology Bulletin, 30(11), 14721484.

Santos, R. F. \& Stein, L. M. (2006). O impacto da emoção na memória e suas distorções: Uma revisão crítica. Porto Alegre: Pontifícia Universidade Católica do Rio Grande do Sul.

Stein, L. M. \& Neufeld, C. B. (2001). Falsas memórias: por que lembramos de coisas que não aconteceram?. Arquivos de Ciência Saúde Unipar, 5(2), 179-186.

Wessel, I., Van der Kooy, P. \& Merckelbach, H. (2000). Differential recall of central and peripheral details of emotional slides is not a stable phenomenon. Memory, 8(2), 95-109.

Recebido em fevereiro de 2007

Reformulado em fevereiro de 2008

Aprovado em abril de 2008

Sobre as autoras:

Carmem Beatriz Neufeld é psicóloga, mestre em Psicologia Social e doutora em Psicologia pela Pontifícia Universidade Católica do Rio Grande do Sul, coordenadora e docente do Curso de Psicologia da Faculdade Assis Gurgacz - Cascavel, PR e avaliadora Institucional e de Cursos de Graduação em Psicologia do INEP/MEC.

Priscila Goergen Brust é psicóloga e mestranda em Cognição Humana no Programa de Pós-Graduação em Psicologia da PUCRS, vinculada ao Grupo de Pesquisa em Processos Cognitivos.

Lilian Milnitsky Stein é psicóloga e mestre em Ciências Cognitivas Aplicadas pelo Ontario Institute for Studies in Education (Canadá), Ph.D em Psicologia Cognitiva pela University of Arizona (EUA), professora adjunta e coordenadora do Grupo de Pesquisa em Processos Cognitivos do Programa de Pós-Graduação em Psicologia da Pontifícia Universidade Católica do Rio Grande do Sul e bolsista Produtividade em Pesquisa - CNPq. 